\title{
JOANNA WARMUZIŃSKA-ROGóż
}

Université de Silésie, Katowice, Pologne

iD https://orcid.org/0000-0001-8195-0099

\section{La traduction littéraire qui n'est pas que la traduction Sur les enjeux traductologiques au Canada}

\author{
Literary translation that is not just a translation \\ About translation issues in Canada
}

\begin{abstract}
The article aims to take a close look at the literary relations between English- and French-speaking Canada. Among different possibilities, the author analyzes specific cases concerning literary prizes, in particular winners of the Governor General's Prize in the category of translation. As it is one of the most prestigious awards in Canada, it undoubtedly conditions the reception of a literary work, being a symbol of prestige. The choice of the jury can sometimes seem surprising or even confusing, as it was the case of Nancy Huston or Jacques Brault who won the prize in the category of translation. Without a doubt, the specificity of the translation à la canadienne goes beyond any distinctions and descriptions and - being also a political issue revolts against all control and all interference.
\end{abstract}

KeY wORDS: literary translation, Canada, literary prize, Nancy Huston, Jacques Brault

Construire des ponts entre le Canada anglais et français, jouer les passeurs de culture, c'est ce que font les traducteurs littéraires dans la plus discrète perfection ${ }^{1}$.

Il va sans dire que la traduction joue un rôle inestimable au Canada, dont témoignent les paroles de Côté, Marcoux et Stratford, rédacteurs du collectif «La traduction littéraire et le Canada $»^{2}$ :

${ }^{1}$ Conseil des arts du Canada. En ligne: www.conseildesarts.ca/cgi-bin/MsmGo.exe?grab $\mathrm{id}=0$ \&page $\mathrm{id}=5310 \&$ query $=$ traducteur\&hiword=TRADUCTEURS $\% 20$ TRADUCTION $\% 20$ TRADUCTIONS\%20TRADUCTRICE\%20traducteur\%20. Date de consultation : le 31 mai 2019.

2 À en croire les rédacteurs, c'est une « conjonction au sens fort» (Côté, MARCoux, STRATFORD 2015 : 134). 
En 1977, Philip Stratford déclarait que le Canada n'avait pas de «tradition» de traduction littéraire, mais qu'une certaine «tendance» en ce sens commençait à poindre, en particulier depuis l'avènement du programme de financement du Conseil des arts du Canada. Près de quarante ans plus tard, il ne fait aucun doute que la traduction littéraire a maintenant une tradition et qu'elle a jusqu'ici joué un rôle-clé dans la formation identitaire du pays, sur les plans culturel, économique, politique, social, etc.

Côté, Marcoux, StratFord 2015: 133

Dans le présent article, nous nous proposons de regarder de près les relations entre le Québec et le Canada anglophone vues par le prisme de la traduction littéraire. Tout d'abord, nous décrirons brièvement un rôle grandissant de la traduction littéraire appuyée par plusieurs démarches institutionnelles pour passer ensuite à un bref aperçu de la spécificité du Prix du Gouverneur général dans la catégorie «Traduction ». Comme c'est l'un des prix les plus prestigieux au Canada, son attribution conditionne sans aucun doute la réception d'une œuvre littéraire dotée de ce symbole de prestige. Parfois, le choix du jury peut sembler quelque peu surprenant voire déroutant comme c'était le cas de Jacques Brault ou de Nancy Huston, tous les deux auteurs et pourtant récompensés pour la traduction. Nous nous attarderons à ces deux cas intéressants ce qui nous permettra d'esquisser quelques traits caractéristiques de la traduction littéraire et de son rôle, détectables au Canada plus qu'ailleurs.

\section{Les enjeux de l'institutionnalisation de la traduction littéraire}

Dans les recherches focalisées sur la spécificité du marché des traductions au Canada on distingue deux périodes, à savoir celle avant l'institutionnalisation de la traduction, soit dans les années 1970, et celle qui commence après ce processus-là. En fait, le changement prend forme avec la Loi sur les langues officielles adoptée par le Parlement canadien en 1969 qui établit officiellement le bilinguisme et qui accorde des droits égaux au français et à l'anglais. La loi a fortement contribué à la promotion de la traduction littéraire tout en déclenchant la création du programme d'aide financière des traductions de la littérature québécoise et canadienne. Puis, en 1971 le gouvernement a lancé la stratégie promouvant le bilinguisme, notamment par le biais du programme d'aide adressé aux éditeurs canadiens qui se décideront à publier les traductions du français vers l'anglais et vice versa. Il va sans dire que - ce que souligne entre autres Jean Delisle - le programme fédéral visait à permettre aux Canadiens de s'ouvrir grâce à la littérature traduite à l'autre solitude et d'en faire la connaissance (Delisle 1998). Ainsi, Le Cercle du Livre de France (qui à présent s'appelle 
les Éditions Pierre Tisseyre), donne en 1973 la vie à la «Collection des Deux Solitudes $»^{3}$.

L'engagement institutionnel dans la traduction littéraire se donne à voir à travers les chiffres. En s'appuyant sur les recherches de Stratford, Lane-Mercier rappelle qu'avant 1960, seulement 16 romans ont été traduits de l'anglais en français et 39 du français en l'anglais (LANE-MERCIER 2014 : 534). Une augmentation significative a lieu dans les années 1972-1985, donc à la suite de la Loi susmentionnée. Par contre, depuis la deuxième moitié des années 1980 on observe un certain ralentissement, voire un affaiblissement du processus : à titre d'exemple rappelons les estimations de Godard selon lesquelles en 1995 on a traduit 16 titres du français vers l'anglais, ce qui constitue la moitié des traductions de 1986 (GoDARD $2000: 477)^{4}$. Ceci découle en partie du fait que le programme du rapprochement des «deux solitudes», si important à l'époque, est devenu en quelque sorte périmé dans la réalité multiculturelle. Ainsi dans le polysystème traductionnel canadien sont apparus d'autres langues et cultures, notamment celles des minorités, des autochtones, des régions et autres. Selon Côté, Marcoux et Stratford,

[e]n outre, on traduit et on publie au Canada un nombre grandissant d'auteurs d'ailleurs, sans compter la popularité des auteurs canadiens plus traduits que jamais. Bref le dialogue littéraire entre les deux langues officielles s'est transformé en un échange pluriel résonnant des langues des Amériques, minoritaires ou non, si bien que le capital littéraire et traductif du Canada a aujourd'hui une portée planétaire. Témoin, l'inauguration du programme de résidence du Centre international de traduction littéraire de Banff, qui accueille chaque été des traductrices et traducteurs littéraires de partout dans le monde.

Côté, Marcoux, Stratford 2015: 134

Or, bien que les démarches traductionnelles se soient déplacées du bilinguisme vers le multilinguisme et le multiculturalisme, le nombre des traductions entre deux langues, le français et l'anglais, reste toujours le plus significatif. Qui plus est, les enjeux concernant les «deux solitudes» s'enracinent profondément dans la tradition et dans le passé canadien.

${ }^{3}$ L'appellation fait penser au titre du roman de Hugh MacLennan Two Solitudes (1945) et symbolise le manque de communication et d'interaction entre les anglophones et francophones, soit entre les « deux solitudes».

${ }^{4}$ Nous nous appuyons uniquement sur des données distinctes ponctuelles en ne disposant pas d'une vue plus générale puisque - comme le fait remarquer LANE-Mercier $(2014: 218)$ - «il n'existe aucun répertoire complet et à jour des œuvres littéraires canadiennes traduites dans les deux langues officielles ». Le dernier ouvrage à ce propos, la Bibliographie de livres canadiens traduits de l'anglais au français et du français à l'anglais de Philippe Stratford a vu le jour en 1977, soit il y a plus qu'une quarantaine d'années. Les données ramassées par la banque de données bibliographiques créée par le Conseil des arts du Canada en 1991 ne concernent que les traductions littéraires subventionnées qui ne représentent ni la totalité des œuvres effectivement traduites ni celles publiées à l'étranger. 


\title{
Les traducteurs littéraires récompensés : le Prix du Gouverneur général
}

\begin{abstract}
À en croire le site «Livres Canada Books » (www.livrescanadabooks.com), au Canada on discerne une trentaine de prix littéraires, sans compter ceux que l'on attribue aux prix jeunesse. À cet égard, le nombre de prix littéraires au Québec sidère: ainsi, sur le site officiel de la Bibliothèque et Archives nationales du Québec on trouve une liste d'une centaine de prix littéraires, parmi lesquels, entre autres, le Prix Athanase-David, pour l'ensemble d'une œuvre, et le Prix Québec-Paris (appelé auparavant le Prix France-Canada), décerné à un écrivain de langue française dont le livre a paru au Canada ou en France. En général, on peut faire la distinction entre deux types de prix au Québec : ceux de portée nationale qui récompensent un livre particulier ou l'ensemble d'une œuvre et, d'autre part, des prix régionaux et locaux, réservés à un groupe d'âge précis ou à un genre concret. Les premiers prix du début du $\mathrm{XX}^{\mathrm{e}}$ siècle visaient surtout à promouvoir le donateur. Ce n'est qu'à partir de 1922 que le gouvernement québécois, à l'initiative d'Athanase David, a instauré plusieurs prix et concours. Une vraie flambée des prix a lieu dans les années 60 du $\mathrm{XX}^{\mathrm{e}}$ siècle et ne cesse de continuer. Indépendamment de leur nombre et type, comme le fait remarquer BORDELEAU, «[c]ontestés parfois, mais convoités toujours, les prix littéraires ont pour but d'assurer la reconnaissance publique de ceux qui font la littérature. Tout en entretenant l'illusion que les lauréats sont forcément les plus méritants » (1994: 18).

D'autre part, il ne faut pas oublier que l'attribution des prix littéraires ne peut pas passer pour objective, dont témoigne le propos de Robert Yergeau:
\end{abstract}

[C]es choix essentiellement arbitraires passent pour objectifs. Si on prenait les prix pour ce qu'ils sont, à savoir des photographies du champ littéraire à un moment précis, la question des jurys et des clans serait moins choquante. Mais un prix sert aussi (sert d'abord?) à assurer le pouvoir symbolique d'un auteur: un lauréat trouve sans peine sa place au sein de l'Institution; en outre, il obtiendra plus facilement des bourses, tandis qu'on le publiera plus volontiers.

cité par BordeleaU 1994: 19

Il va sans dire que parmi les prix littéraires ce sont les Prix du Gouverneur général qui occupent une place particulière parmi d'autres prix au Canada et au Québec. Ils ont été fondés en 1936 par le gouverneur général John Buchan, Lord Tweedsmuir, et la Canadian Authors Association établie en 1921. Il faut souligner que le prix ne sera attribué à un ouvrage en langue française qu'en 1959 et ceci, à en croire Irvine, "en raison de défis organisationnels » (IRVINE 2018 : 37). À partir de 1959, c'est le Conseil des arts du Canada, qui est responsable 
du financement, de l'administration et de la promotion. Les Prix littéraires du Gouverneur général sont attribués aux ouvrages de langue française et de langue anglaise dans chacune des sept catégories suivantes : romans et nouvelles, essais, poésie, théâtre, littérature jeunesse - texte, littérature jeunesse - livres illustrés et, à partir de 1987, traduction (anglais et français). Comme nous lisons sur le site web:

Les Prix font la promotion de la littérature canadienne afin d'inciter les Canadiennes et les Canadiens à la lecture. Ils procurent aussi aux œuvres des finalistes et des gagnants une reconnaissance de leurs pairs et de la population: Allez! Lisez nos meilleurs livres! $!^{5}$

Une petite curiosité : il arrive que le même ouvrage obtienne le prix deux fois - pour la première fois, dans sa catégorie respective, et pour la deuxième fois, dans la catégorie traduction. Ainsi, François-Marc Gagnon obtient en 1978 avec son ouvrage Paul-Emile Borduas (1905-1960) le prix dans la catégorie «Études et essais», et puis, en 2014 c'est la traduction par Peter Feldstein du même ouvrage qui est récompensée dans la catégorie "Traduction» (cf. IRvine 2018).

Au total, dans les années 1936-2017, 31 livres ont été primés dans la catégorie «Traduction» du français vers l'anglais et autant en sens inverse. Nous n'analyserons pas ici en détail les listes de traductions primées, vu leur nombre et le but de notre article 6 . Ce qui nous intéresse le plus, ce sont deux cas particuliers évoqués, celui de Huston et de Brault, qui permettront de problématiser la question de la traduction littéraire au Canada.

\section{La traduction, l'auto-traduction, la réécriture? Les controverses autour du roman de Nancy Huston}

«Albertaine défroquée », «anomalie territoriale», «Anglaise récalcitrante» (Huston 1995 : 264), et par dessus tout, écrivaine canadienne et française, mais non pas canadienne-française, voici quelques-uns des épithètes donnés à Nancy Huston après l'attribution du Prix du Gouverneur général du Canada à l'écri-

\footnotetext{
${ }^{5}$ https://livresgg.ca/a-propos. Date de consultation: le 2 juin 2020.

${ }^{6}$ Pour plus de détail concernant les lauréats du Prix du Gouverneur général, on peut notamment consulter l'opuscule de Andrew David Irvine qui a fait un travail de bénédictin et a ramassé la liste de tous les prix et auteurs dès le début jusqu'en 2017 (cf. IRvine 2018).
} 
vaine pour son auto-traduction Cantique des plaines en 19937. L'« affaire Nancy Huston » est liée à la controverse quant à l'appartenance de l'œuvre récompensée à la catégorie «Romans et nouvelles $»^{8}$. Premièrement, on dit que le prix a été décerné à la traduction et non pas à une œuvre originale. En deuxième lieu, les adversaires, parmi lesquels cinq éditeurs montréalais, ont soulevé l'argument qu'il s'agit d'un auteur qui n'est ni québécois ni francophone.

L'écrivaine elle-même ne partage pas l'opinion des critiques, en répondant notamment: «Je revendique le fait d'être l'auteur des deux versions, c'est tout» (citée par Wilhelm 2006: 75). De plus, à en croire l'auteure, le Cantique des plaines est une deuxième version originelle du même livre ce qu'elle souligne entre autres dans sa lettre adressée au Conseil des Arts du Canada (cf. WILHELM 2006: 75). L'auteure insiste sur le fait qu'il s'agit de la réécriture et que, comme le rappelle pertinemment Potvin, «la version française l'avait même amenée à retravailler le texte anglais » (Potvin 1997: 9). Il s'agit donc d'un processus d'aller-retour entre les deux langues.

Il convient aussi de revenir au deuxième argument des adversaires de Nancy Huston, à savoir sa nationalité. Comme le rappelle Mossière,

dans son entrevue avec Stéphan Bureau, Nancy Huston tombe singulièrement d'accord avec ceux qui ont critiqué l'attribution du Prix du Gouverneur général à son roman, en remarquant que ce prix lui avait été attribué par des destinataires auxquels il n'était nullement adressé et qui, pouvons-nous désormais ajouter, en étaient partiellement exclus d'un point de vue linguistique.

Mossière 2007 : 94

Il n'empêche que le prix, mais aussi le scandale qui se lie avec lui, a permis de faire connaître Nancy Huston et son œuvre parmi les lecteurs québécois auxquels, paradoxalement, elle n'a pas adressé le Cantique des plaines ${ }^{9}$.

Rappelons que l'auteure commence sa carrière en écrivant en français qui l'a libérée de l'anglais, soit sa langue maternelle, et de son passé canadien-anglais. Ainsi, comme dans le cas d'un nombre non négligeable des écrivains, «la langue seconde [...] ouvre [pour elle] les sentiers de la création» (KLEINLATAUd 1996: 213). Puis, après les premiers succès littéraires en France, et

${ }^{7}$ Par ailleurs, en 1996, Nancy Huston a obtenu de nouveau une nomination pour le même prix pour son roman intitulé Instruments des ténèbres.

${ }^{8}$ Il convient de citer le nom de Daniel Gagnon qui, lui aussi, s'est vu attribuer le prix, dans son cas c'était le Prix Molton de l'Académie des lettres au Québec, pour son roman La fille à marier (Leméac 1985), écrit tout d'abord en anglais sous le titre The Marriageable Daughter et jamais publié en version originale.

${ }^{9}$ Gilles Mossière a proposé une analyse intéressante de la langue française que Huston utilise dans le Cantique de plaines, qui, selon le chercheur, est plus approprié aux lecteurs français de France, mais qui, par contre, semblent être moins initiés aux questions culturelles que l'écrivaine aborde (cf. Mossière 2007). 
ailleurs, Huston redécouvre en quelque sorte l'anglais tout comme elle a découvert le français au début de sa carrière. Il s'agit donc de s'apprivoiser une langue tout d'abord étrangère. Une fois le processus initié, l'écrivaine se sent prête à revenir à l'anglais :

Avec ce livre [Plainsong], mes racines ont pris de l'intérêt pour moi. J'avais toujours dit à tout le monde que je venais d'un pays plat, avec une histoire inexistante, une culture zéro. Et peu à peu, je me suis aperçue qu'il pouvait y avoir de la passion, de la magie et de la matière littéraire dans mes racines. Et ça m'est venu en anglais. J'entendais la musique de l'anglais. Des cantiques, des chansons de cow-boy, et de travailleurs des chemins de fer. Il fallait que ce soit en anglais.

Huston dans l'interview avec LAURIN 1993

Les enjeux langagiers propres à l'écriture et à la réécriture ${ }^{10}$ ou la re-création hustonienne encouragent une réflexion sur l'appartenance de la littérature à un espace national concret. Ainsi, Jean Royer est d'avis que le choix de l'anglais comme la langue de Plainsong rédigé en tant que premier positionne Huston comme une écrivaine canadienne-française (Royer, dans SimON 1994 : 49). Or, il est à noter que les romans précédents ont été rédigés en français ce qui rend l'argument de Royer injustifiable. De plus, Huston pratique parfois l'écriture à deux voix, c'est-à-dire elle alterne le français et l'anglais dans le même texte, comme dans le manuscrit d'Instruments des ténèbres où les deux langues se trouvent côte à côte. Comme le rappelle Mossière: "Canadienne-anglaise écrivant en français à Paris sur le Canada anglais, Nancy Huston a souvent dit qu'elle écrit dans le fossé entre les cultures, dans la rupture» (Mossière 2007: 98-99). Par ailleurs, nombreux sont ceux qui défendent la non-appartenance stricte à une culture et une langue de Nancy Huston. Ainsi, Régine Robin, parmi beaucoup d'autres, s'insurge contre "l'impossibilité à penser la non-coïncidence de soi à soi, de l'écart entre le territoire, la culture, la langue et les modalités multiples de l'identité» (RoBIN 1994 : 3).

La réécriture hustonienne mise en pratique dans le Cantique de plaines et la controverse autour du Prix du Gouverneur général attribué à ce roman incitent aussi à réfléchir sur la définition de l'œuvre traduite. Selon Sherry Simon, en premier lieu, il faudrait repenser les frontières entre l'écriture en langue originale et la version en langue étrangère. La chercheuse ne partage pas l'opinion de Jacques Allard selon qui « [q]uand on raconte la même histoire, en passant d'une langue à une autre, il est évident que, quelle que soit la recréation langagière (et le Cantique est bellement écrit), l'on raconte une histoire déjà racontée » (Allard, dans Simon 1994 : 48). Selon Simon, « comment savoir où est l'avant et l'après, la

${ }^{10}$ KLein-Lataud souligne que Huston insiste sur le terme de réécriture pour son procédé (1996 : 220). 
langue première et la langue seconde, l'identité véritable et l'identité d'emprunt, l'original et la traduction?» (1994: 49). Il semble que Huston partage les doutes de Sherry Simon. Ainsi, dans son essai «Traduttore non è traditore», paru dans le recueil collectif Pour une littérature-monde, l'auteure de Plainsong soutient que l'écrivain «écrit pour agrandir le monde, pour en repousser les frontières. Il écrit pour que le monde soit doublé, aéré, irrigué, interrogé, illuminé par un autre monde, et qu'il en devienne habitable. Ce faisant, l'écrivain traduit. Ce n'est jamais chose facile. On fait ce qu'on peut» (Huston 2008 : 153-154).

\section{La traduction qui se mélange avec l'écriture : le cas de Jacques Brault}

Transfiguration est un recueil poétique paru en 1998 simultanément chez Noroît et BuschekBooks. Jacques Brault le compose en duo, avec E.D. Blodgett, et c'est la deuxième fois qu'il travaille ainsi (pour la première fois il a co-écrit en 1993 le recueil intitulé Au petit matin avec Robert Melançon).

E.D. Blodgett, un poète, traducteur et critique littéraire canadien, et Jacques Brault, un poète francophone, se donnent pour tâche de composer un dialogue poétique qu'ils décrivent en détail dans les introductions : «Prefatory Note» de Blodgett et «Liminaire» de Brault. Il s'agit donc de «[p]oèmes dialogants ou poèmes à deux voix» (BlODgetT, Brault 1998: 9), autrement dit d'un poème inspiré du renga japonais ou plusieurs poètes composent un seul poème en suivant des règles précises. Or, Blodgett et Brault, tout en s'inspirant du projet japonais, ne suivent pas de règles strictes, mais se limitent plutôt au principe de composer de concours un seul poème, avec cette restriction que «[c]hacun a écrit dans la langue et selon sa dictée propre, mais en répondant à l'autre par l'écriture oblique du poème» (1998: 9). Les chercheurs constatent à voix unanime qu'il ne s'agit pas de traduction, mais plutôt d'un dialogue dans lequel les deux versions se répondent (Suchet 2017: 5). Ainsi la traduction suit toujours l'original, et puis c'est la traduction qui se transforme en un texte original traduit à son tour. Sur chaque page, il y a donc l'original qui constitue un texte source (engendrant la suite dans l'autre langue) et en même temps un texte cible (en tant que la réponse au fragment précédent). Les originaux proprement dits et les traductions se chevauchent ainsi et les traductions se transforment imperceptiblement en originaux (LECLERC 2014: 497). Il va sans dire que le recueil de Blodgett et Brault constitue un projet original non seulement dans le contexte canadien où le contact entre les deux cultures, anglo- et francophone est toujours problématique, mais aussi dans un contexte universel. De plus, il s'agit ici d'une vision tout à fait originale de la création littéraire et de la traduction qui est élevée 
au même niveau. C'est par ailleurs le titre même du recueil qui reflète cette idée. Blodgett l'explique dans son introduction de manière suivante :

each dancer must surrender to the other to create the figure that the dance, not the dancers, requires. And so we have chosen to call this renga "transfiguration" to suggest how each dancer, while remaining himself, is drawn into the figure that possesses both dancers that becomes one dancer, whom they did not imagine.

Blodgett, dans Blodgett, Brault 1998: 8

Brault caractérise le recueil ainsi forgé comme suit: «Si la poésie est aussi la voix de l'autre en soi-même, alors ce petit livre constitue peut-être, et à maints égards, une transfiguration » (Brault, dans Blodgett, Brault 1998: 9). Or, ce projet créateur obtient en 1999 le Prix du Gouverneur général - chose surprenante - dans la catégorie «Traduction », ce qui est, d'une certaine manière, l'acte de sanctionner la présence de la traduction proprement dite au sein de l'œuvre. Ce qui est encore plus surprenant, c'est que c'est uniquement Jacques Brault qui en est lauréat en tant que traducteur de l'anglais vers le français. Comme le fait remarquer Leclerc, «Transfiguration [est] ainsi ramenée aux catégories préconstruites d'œuvre originale (anglaise) et de traduction (française)» (LeCLERC 2014 : 497).

La décision du jury est d'autant plus déconcertante que le recueil de Brault et de Blodgett est conçu comme un dialogue à deux voix (et deux langues) dans lequel la frontière entre ce qui est original et ce qui est traduit, s'estompe, et - qui plus est - tout ce qui est secondaire constitue un point de départ pour l'écriture proprement dite. De plus, comme le fait remarquer Leclerc, «[1]eur renga translingual se construit précisément contre la hiérarchie habituelle entre original et traduction, entre auteur et traducteur» (LeCLERC 2014 : 501). Sherry Simon le voit ainsi :

This is reciprocal writing taken to the furthest point, where words never entirely belong to a single speaker. As the dialogue advances, there is a progressive interconnection between the poets' voices. Transfiguration illustrates the desire to exploit translation's potential to scramble ownership and property. Rather than maintaining separation between languages and identities, these versions shuffle them. The voice of each poet is penetrated by the accent, the vocabulary, the sensibility of the other.

SIMON 2006: 140-141

De fait, l'attribution du prix dans la catégorie «Traduction» à Brault, ne serait-ce pas une incompréhension totale de l'idée du poète? C'est Brault qui a écrit que «nous [les Québécois] n'aimons ni traduire ni être traduits» car «[1]es clefs de la traduction appartiennent aux puissants», sans oublier qu'il y a des langues colonisatrices «et nous l'éprouvons durement, chaque jour» 
(BRAult 1975 : 16). Pour pallier à cette inégalité Brault recourt à des pratiques déviantes comme la «nontraduction» dans les Poèmes de quatre côtés, ou comme l'«inter-texte», «un texte, ni d'autre, ni de moi» (BRAUlt 1975: 50) dans Transfiguration. Leclerc voit dans ce projet original, bilingue, une critique de la politique canadienne linguistique qui repose sur le bilinguisme officiellement décrété et qui rend la traduction de l'anglais vers le français et vice versa obligatoire (LECLERC 2014 : 497). Selon Sherry Simon, c'est plus que la critique, c'est la parodie même du bilinguisme officiel, bien que Transfiguration soit libre des accents explicitement politiques (SImON 2006: 140). De plus, la chercheuse fait remarquer que

[1]e trouble qu'évoque Jacques Brault dans sa relation aux langues n'est pas que poétique. Il est aussi profondément ancré dans le contexte historico-politique du Québec. Jacques Brault est depuis toujours un critique attentif aux rapports de force qui jouent sur la scène de l'écriture. Comment faire l'éloge de la traduction dans le contexte de la «surconscience» linguistique du Québec?

SimON 1994: 63

\section{En guise de conclusion}

De ce qui précède découlent deux conclusions. En premier lieu, il va sans dire qu'au Canada, la traduction, y compris la traduction littéraire, est intrinsèquement liée à l'identité même, dont témoigne notamment le titre du collectif cité au début de notre présentation: «La traduction littéraire et le Canada». Ses rédacteurs expliquent qu'ils voulaient

établir un lien clair entre la traduction littéraire et le Canada (conjonction au sens fort) qui invite à la penser comme une constituante de la singularité canadienne, un lieu de synthèse de diverses influences, prolongement des cultures européennes, métissage avec les peuples autochtones, pluralité de langues, en plus des langues officielles. Le Canada est un pays traducteur s'inscrivant dans un continent traducteur. Par conséquent, il est intéressant de le lire comme l'héritier de traditions intellectuelles dont l'originalité est le fruit de la traduction.

Côté, Marcoux, Stratford 2015: 134

À cet égard, il ne faut pas oublier que cette liaison tire sa puissance en grande partie de l'appui institutionnel. Lane-Mercier le résume de manière suivante: « la traduction institutionnelle fait partie intégrante de nos droits fondamentaux comme de notre identité nationale et de notre quotidien» (LANE-Mercier 2014 : 519). Or, l'engagement des autorités, que ce soit au niveau provincial ou fédéral, 
aussi louable soit-il, sera toujours et inévitablement politique. Quant au Québec, il s'agit avant tout de la promotion de la littérature québécoise hors le Québec, c'est un objectif qui est propre entre autres au programme d'aide à la traduction de la SODEC:

Au Québec, le programme d'aide à la traduction de la SODEC actuellement en vigueur vise deux objectifs: soutenir la traduction d'œuvres littéraires québécoises, stimuler l'exportation et offrir une plus grande visibilité de notre littérature à l'extérieur du Québec. Des subsides sont offerts pour la "traduction d'un auteur québécois, du français ou de l'anglais vers l'une ou l'autre de ces deux langues, ou vers toute autre langue ».

Desmeules 2015

Quant au niveau fédéral, Desmeules observe que

le Conseil des arts du Canada n'accorde de son côté que des subventions pour la traduction - en français, en anglais ou dans une langue autochtone "d'une cuvre littéraire d'un écrivain canadien en vue de sa publication au Canada ». Le Programme national de traduction pour l'édition du livre semble à cet égard être asservi à la stratégie du gouvernement du Canada en matière de langues officielles.

Desmeules 2015

Cet appui de la part de l'État change à un tel point le paysage traductionnel du pays qu'il serait impossible d'analyser le pôle littéraire dans le contexte canadien à l'aide des outils proposés par Pierre Bourdieu. À en croire Barbara GODARD, «[c]ette intervention gouvernementale directe structure deux marchés superposés : un modèle national subventionné coexiste avec un marché capitaliste soumis aux contraintes économiques internationales » (2002: 75).

Deuxièmement, la spécificité de la traduction au Canada, de son rôle dépassant largement une simple transmission du message d'une langue à l'autre, fait qu'il est impossible de l'ajuster à des définitions nettes et bien établies. Sherry Simon l'explicite ainsi :

In both cases, I would like to emphasize a shift in the mandate which Canadian translation seems to be giving itself. When Philip Stratford in the 1970s called translations of Quebec literature "news from the front," he was referring to an intense flurry of exchange between Quebec and English Canadian literature, an exchange motivated by curiosity and fascination. Translators were called upon to "transmit" Quebec literary news to new readers. The informative and ambassadorial role, I would argue, is now being augmented by another dimension. Rather than acting exclusively as mediators, writer/translators are increasingly involved in creating hybrid literary texts which are informed by a double culture. The novels of Gail Scott, the "renga" of Brault and Blodgett 
are examples of such texts. They use languages to cross traditions, making their texts a crossroads of sensibilities.

SimON 2000: 74

Ceci dit, il semble que les deux cas particuliers décrits plus haut, celui de Nancy Huston et de Jacques Brault et des Prix du Gouverneur général, reflètent on ne peut mieux la spécificité de la traduction «à la canadienne» qui échappe à des distinctions et descriptions et qui - tout en étant une question politique - se révolte contre tout contrôle et toute ingérence.

\section{Bibliographie}

Blodgett E.D., Brault, Jacques, 1998: Transfiguration. Saint-Hippolyte (Québec) et Toronto, Noroît et BuschekBooks.

Bordeleau, Francine, 1994: «Les prix littéraires: l'ère du soupçon». Lettres québécoises : la revue de l'actualité littéraire, 75 , pp. 18-21.

Brault, Jacques, 1975 : Poèmes des quatre côtés. Québec, Éditions du Noroît.

CôtÉ, Nicole, Marcoux, Danièle, Stratford, Madeleine, 2015: «La traduction littéraire et le Canada : présentation». TTR, 28 (1-2), pp. 133-138. https://doi.org/10.7202/1041653ar. Date de consultation: le 20 septembre 2019.

Delisle, Jean, 1998: «Traduction au Canada: Survol historique (depuis 1534)». (sans date). En ligne. https://www.academia.edu/5940734/La traduction au Canada survol historique depuis_1534 (date de consultation: 9.05.2016). Ce texte est paru en version anglaise sous le titre «Canadian Tradition» dans Mona BAKER (dir.) : Routledge Encyclopedia of Translation Studies. Londres / New York, Routledge, 1998, pp. 356-363.

Desmeules, Christian, 2015: «Traduire le monde». Le Devoir, 14 novembre. En ligne. https:// www.ledevoir.com/lire/455213/traduire-le-monde. Date de consultation: le 20 septembre 2019.

GODARD, Barbara, 2000: «French-Canadian Writers». In: Encyclopedia of Literary Translation into English. Vol. 1. O. Classe (dir.). London-Chicago, Fitzroy Dearborn Publishers, pp. $480-481$.

GODARD, Barbara, 2002: «La traduction comme réception: les écrivaines québécoises au Canada anglais ». TTR: traduction, terminologie, rédaction, 15.1, pp. 65-101.

Huston, Nancy, 1995: A Tongue Called Mother. Désirs et réalités. Textes choisis 1978-1994. Montréal, Leméac.

Huston, Nancy, 2008: "Traduttore non è traditore». In : Jean RouAud et Michel Le BRIS, dir. Pour une littérature-monde. Paris, Gallimard, pp. 151-160.

Invine, Andrew David, 2018: Les Prix littéraires du Gouverneur général du Canada: Une bibliographie (English and French Edition). Ottawa, University of Ottawa Press.

Klein-Lataud, Christine, 1996: "Les voix parallèles de Nancy Huston». TTR, 9 (1), pp. 211-231.

Lane-Mercier, Gilian, 2014 : «Les carences de la traduction littéraire au Canada : des bibliographies et des traditions ». Meta, 59(3), pp. 517-536. https://doi.org/10.7202/1028655ar. Date de consultation: le 20 septembre 2019. 
LaUrin, Danielle, 1993 : «Source sûre, interview de Nancy Huston». Voir, 16-22 septembre.

LeClerc, Catherine, 2014: «Bilinguisme officiel et traduction au Canada: les interprétations littéraires de Patrice Desbiens et de Jacques Brault / E.D. Blodgett». Meta, 59 (3), pp. 494-516.

Mossière, Gilles, 2007: «Lecture(s): exclusion et altérité dans Cantique des plaines de Nancy Huston ». Cahiers franco-canadiens de l'Ouest. Vol. 19, n 1, pp. 91-103.

Potvin, Claudine, 1997: «Inventer l'histoire: la plaine revisited». Francophonies d'Amérique, 7, pp. $9-18$.

Robin, Régine, 1994 : «Speak Watt. Sur la polémique autour du livre de Nancy Huston». Spirale, avril.

Simon, Sherry, 1994 : Le trafic des langues : traduction et culture dans la littérature québécoise. Montréal, Boréal.

Simon, Sherry, 2000: «The Paris Arcades, the Ponte Vecchio and the Comma of Translation». Meta, 45, $\mathrm{n}^{\circ} 1$, pp. 73-79.

Simon, Sherry, 2006: Translating Montreal: Episodes in the Life of a Divided City. Montréal \& Kingston, McGill-Queen's University Press.

SUCHET, Myriam, 2017: «Jacques Brault et la nontraduction, un Unland original», TRANShttp://journals.openedition.org/ trans/1646. Date de consultation: le 20 avril 2018.

WiLhelm, Jane Elisabeth, 2006: «Autour de Limbes/Limbo: un hommage à Samuel Becket de Nancy Huston ». Palimpsestes, 18, pp. 59-86.

Conseil des arts du Canada. En ligne. www.conseildesarts.ca/cgi-bin/MsmGo.exe?grab $\mathrm{id}=0 \&$ page_id=5310\&query=traducteur\&hiword=TRADUCTEURS\%20TRADUC TION\%20TRADUCTIONS\%20TRADUCTRICE\%20traducteur\%20. Date de consultation: le 31 mai 2019.

www.livrescanadabooks.com. Date de consultation: le 31 mai 2019.

\section{Notice biographique}

Joanna Warmuzińska-Rogóż est docteure habilitée à diriger les recherches, professeure à l'Institut d'Études littéraires de l'Université de Silésie. L'auteure de deux monographies (De Langlois à Tringlot. L'effet-personnage dans les Chroniques romanesques de Jean Giono - analyse sémiopragmatique, 2009 ; Szkice o przekładzie literackim. Literatura rodem z Quebecu w Polsce, 2016 Prix Pierre Savard), co-rédactrice du $3^{\mathrm{e}}$ numéro de TransCanadiana (2010) et du $13^{\mathrm{e}}$ numéro de Romanica Silesiana (2018), co-auteure, avec Krzysztof Jarosz, de Antologia współczesnej noweli quebeckiej (2011) et auteure de nombreux articles sur la littérature québécoise et la traduction littéraire.

joanna.warmuzinska-rogoz@us.edu.pl 